Nuclear Physics B144 (1978) 525-535

(c) North-Holland Publishing Company

\title{
WILL LARGE WEAK INTERACTION EFFECTS BE OBSERVABLE AT VERY HIGH ENERGIES? *
}

\author{
H.E. HABER and G.L. KANE \\ Physics Department, University of Michigan, Ann Arbor, MI 48109
}

Received 2 June 1978

We examine the possibility that some weak interaction cross sections become large at very high energies. Such effects could lead to observable weak interaction phenomena in very high energy pp and $p \bar{p}$ colliding beams. Apart from direct production of W's and Z's, the possibility of observable weak effects requires the Higgs mass to be much larger than the mass of the vector bosons. However, for feasible experiments such effects are suppressed in many popular gauge theories, and we expect that observable weak cross sections will not get large at high energies. Theories with more than one Higgs boson may allow large observable effects.

\section{Introduction}

At low energies, weak interaction total cross sections rise linearly with lab. energy. The phenomenological four-fermion coupling gives an s-wave interaction which grows to violate the unitarity limit at c.m. energies of order $1 / \sqrt{ } G_{\mathrm{F}} \simeq 300 \mathrm{GeV}$. Because of this situation, it is commonly stated that weak cross sections will get large at very high energies, comparable with strong interactions.

In the context of gauge theories the situation becomes more precise. It has been discussed most thoroughly by Veltman $[1,2]$, in terms of two thresholds. In any case one expects massive gauge vector bosons, charged $W^{ \pm}$'s and neutral $Z^{0}$ 's. These eliminate the point interaction and give finite $\nu$ p total cross sections, for example. They will also be produced directly, giving large cross sections in $\mathrm{e}^{+} \mathrm{e}^{-}$collisions when the c.m. energy equals the $Z^{0}$ mass, and enhancements in $\nu p \rightarrow Z^{0}+\ldots, p p \rightarrow$ $\mathrm{Z}^{0}+\ldots$, etc. Their mass range constitutes the first threshold.

But the theory with only the massive vector bosons is not renormalizable, and additional physics must enter. In the conventional versions this occurs through the Higgs bosons. To observe experimentally (and test) the features which make the theory renormalizable, and to test particular gauge theories, one must observe effects due to the additional physics. If the Higgs boson mass is large compared to

* Research supported in part by the Department of Energy. 
$m_{\mathrm{W}}$ it gives the second threshold [1-3]. (If the Higgs boson mass is small (e.g. less than $m_{\mathrm{W}}$ ), it will have to be observed directly to see any effects.)

Veltman [2] has studied the possibility of testing the gauge theories at low energies, and found that it is not easy. In the present paper we discuss whether some observable effects may be expected at high energies. Unfortunately, the answer is probably negative.

We can understand this as follows. In the conventional gauge theories the new physics enters at high energies essentially by replacing a factor of $s$, which would lead to a large amplitude, by $m_{\mathrm{H}}^{2}\left(m_{\mathrm{H}}\right.$ is the Higgs boson mass). Veltman [1], and Lee, Quigg and Thacker [3], have observed that there are then two situations. If $m_{\mathrm{H}}$ is less than or comparable to $m_{\mathrm{W}}$ and $m_{\mathrm{Z}}$, then all weak amplitudes stay rather small in all circumstances and higher-order effects which will test the structure of the theory are essentially undetectable. However, if $m_{\mathrm{H}}$ is large, so that some weak amplitudes are large, then higher-order effects are large and perhaps some are observable.

Both from the requirements that radiative connections were of order unity, and from the unitarity limit, it was argued $[1-4]$ that perturbative calculations would break down and weak amplitude would behave strongly for $m_{\mathrm{H}} \sim 10^{3} \mathrm{GeV}$. Unfortunately, when one wishes to observe such effects experimentally at high energies one must begin with fermion states, e.g. in $\mathrm{e}^{+} \mathrm{e}^{-}$collisions or in $\mathrm{q} \overline{\mathrm{q}}$ collisions (where the quarks are in $p$ or $\bar{p}$ beams). Thus, when one draws the appropriate Feynman diagrams, one finds in general that the Higgs lines attach to fermions. Since Higgs particles with non-zero vacuum expectation values will contribute to the fermion mass, their coupling strength is proportional to the fermion mass, $m_{\mathrm{f}}$. In fact, the corrections due to higher order effects in observable processes turn out to be at most of order:

$$
g^{4}\left(m_{\mathrm{f}} / m_{\mathrm{W}}\right)^{2} \ln m_{\mathrm{H}}^{2} .
$$

This is numerically negligible since $m_{\mathrm{f}} / m_{\mathrm{W}} \lesssim 10^{-3}$. We conclude that within the framework of the simplest gauge theories, with scalar Higgs mesons, and fermion masses determined in part by coupling to the Higgs particles, no large high energy weak interaction amplitudes will be observed, apart from those directly due to production of W's and Z's. In the case of gauge theories with more complicated Higgs structures, such conclusions are not immediate. Indeed, some gauge theories [5-7] suggest that the Higgs-fermion coupling need not always be small. If large weak effects not associated with W's and Z's are found, one will have evidence that $m_{\mathrm{H}}$ is large, perturbative arguments are not useful, and a Higgs-fermion coupling exists which is not proportional to $m_{\mathrm{f}} / m_{\mathrm{W}}$.

We have mentioned that in order to observe large weak interactions, one must begin with fermions. Eq. (1) was the first correction to $f f \rightarrow$ ff scattering. We have also looked at $\mathrm{f} \overline{\mathrm{f}} \rightarrow \mathrm{W}^{+} \mathrm{W}^{-}$where the Higgs plays an important role. Again, we find the appearance of the factor $\left(m_{\mathrm{f}} / m_{\mathrm{W}}\right)^{2}$ which makes the Higgs contribution to the high energy cross section negligible. 
In sect. 2 we give the details of the analysis that shows the presence of the $\left(m_{\mathrm{f}} / m_{\mathrm{W}}\right)^{2}$ factor which makes higher-order contributions numerically negligible. In sect. 3 we remark on theories which could give large effects at very high energies, and in sect. 4, we briefly discuss the production of two $\mathrm{W}$ bosons.

\section{Analysis of fermion-fermion scattering including loop corrections}

Recently Veltman [1,2], and Lee, Quigg and Thacker [3], have discussed in detail the role of the Higgs boson in the dynamics of high-energy weak interactions. If $m_{\mathbf{H}}$ is of order $1 \mathrm{TeV}$, weak interactions in the boson sector become strong and perturbative arguments break down. If $m_{\mathrm{H}}<<1 \mathrm{TeV}$, weak amplitudes do not become large. We analyze $\mathrm{f} \overline{\mathrm{f}}$ (or $\mathrm{ff}$ ) scattering because to study weak interactions experimentally one must begin with fermions (quarks or leptons). Since the value of $m_{\mathrm{H}}$ affects the size of the amplitudes, and amplitudes are small when $m_{\mathrm{H}}$ is small, we concentrate on the large $-m_{\mathrm{H}}$ limit.

One could hope that loop corrections to ff scattering would be large enough to detect at very high energies. For example, in the contributions of fig. $1 \mathrm{a}-\mathrm{c}$ the terms of order $s$ in the amplitude cancel, but a growing term of order $\sqrt{ } s$ is left which must be cancelled by the Higgs contribution of fig. $1 \mathrm{~d}$ to give a renormalizable theory $[4,8,9]$. Folding these back on themselves to give a final fermion with virtual bosons in loop integrals might give effects of order $m_{\mathbf{H}}^{2}$. Veltman's analysis of a massive Yang-Mills theory without a Higgs mechanism reinforces this expectation; to make the theory finite a cut-off is used, and the size of the higher-order corrections grows as the cutoff does (except that $\ln m_{\mathrm{H}}^{2}$ rather than $m_{\mathrm{H}}^{2}$ appears in the

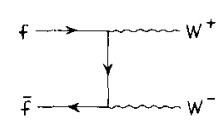

(a)



(b)

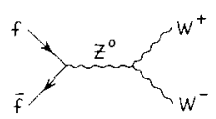

(C)

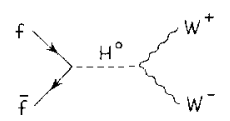

(d)

Fig. 1. Tree diagrams for $\mathrm{f} \overline{\mathrm{f}} \rightarrow \mathrm{W}^{+} \mathrm{W}^{-}$in the Weinberg-Salam model. 
leading term). But it turns out that quite generally the growing terms have a factor of $\left(m_{\mathrm{f}} / m_{\mathrm{W}}\right)^{2}$ and are numerically negligible for experiments.

Here we describe briefly how to see the origin of the $\left(m_{\mathrm{f}} / m_{\mathrm{W}}\right)^{2}$ factor in the conventional gauge theories with a Higgs mechanism. Consider for simplicity an SU(2) gauge theory with one Higgs doublet (the results are similar for the Weinberg-Salam model [10]). We need to examine higher order corrections to ff scattering when $m_{\mathrm{H}}^{2}$ is large. For a complete analysis, we would have to do the renormalization through the one-loop level. This is a complicated procedure for spontaneously broken gauge theories, since there are many counterterms which are all interrelated. However, for our purposes here, we need only consider induced terms at the one-loop level. Since the theory is renormalizable, these terms will be finite. For example, consider the Wf $\bar{f}$ vertex. In the $S U(2)$ gauge theory, the coupling is of the form $\frac{1}{8} i g \gamma_{\mu}\left(1+\gamma_{5}\right) \tau_{a}$; that is, the coupling is pure $\mathrm{V}-\mathrm{A}$. Therefore, any induced $\mathrm{V}+\mathrm{A}$ term appearing in higher orders of the perturbation series must be finite.

In this section, all calculations are performed in the 't Hooft gauge. The relevant Feynman rules for the SU(2) gauge theory in the 't Hooft gauge are given by Veltman in ref. [1]; the physical and ghost particles existing in this gauge are depicted in fig. 2. The Feynman diagrams which contribute to the Wff vertex correction at the one-loop level are given in fig. 3 . Let us look at pieces proportional to $\gamma_{\mu}\left(1-\gamma_{5}\right) \tau_{a}$ We calculate these diagrams using the dimensional regularization technique [11]. Now we might find for an individual graph a term proportional to $(n-4)^{-1}$ (where at the end of the calculation we take $n \rightarrow 4$ ). However, we are assured by the renormalizability of the theory that all such pieces cancel out when the diagrams of fig. 3 are added up. Therefore, we simply discard these terms and analyze those graphs which give large contributions from $m_{\mathrm{H}} \rightarrow \infty$. We have found that the only one-loop graphs involved in ff scattering which become large as $m_{\mathrm{H}} \rightarrow \infty$ must satisfy one of the following conditions.

(i) If the loop integral is convergent, then the loop must contain exactly one physical Higgs $(H)$ line which is connected to two ghost Higgs $(\chi)$ lines. (The Feyn-

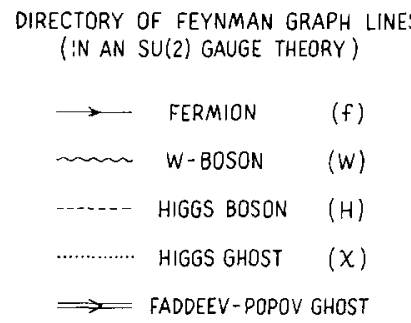

Fig. 2. Possible Feynman graph lines in the 't Hooft gauge in an SU(2) gauge theory. The Wboson and Higgs ghost $(x)$ can have charges \pm 1 or 0 . The fermions can have charges -1 or 0 . The physical Higgs (H) and Faddeev-Popov ghost are neutral. 


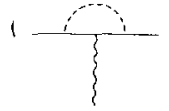

(a)

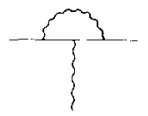

(d)

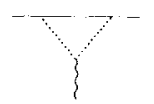

(g)



(b)

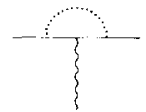

(e)

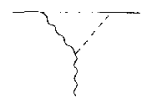

(h)

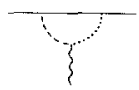

(c)

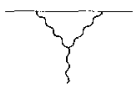

(f)

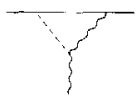

(i)

Fig. 3. The Wff vertex correction. We have drawn the one-loop Feynman diagrams which contribute to the Wff vertex correction. See fig. 2 for a definition of the various lines.

man rules provide an extra factor of $m_{\mathrm{H}}^{2}$ here.) Such a graph will be proportional to $\ln m_{\mathrm{H}}^{2}$.

(ii) If the loop integral is logarithmically divergent, the loop must contain exactly one physical Higgs line. Such a graph will also be proportional to $\ln m_{\mathrm{H}}^{2}$. All other radiative corrections (which could introduce factors of $m_{\mathrm{H}}$ ) result in the redefinition of the physical parameters of the theory (e.g. corrections to propagators) and are thus not observable. Consequently, at the one-loop level in the limit of $m_{\mathrm{H}} \rightarrow \infty$, only terms proportional to $\ln m_{\mathrm{H}}^{2}$ appear. We now catalogue all vertex corrections which contribute a factor of $\ln m_{\mathrm{H}}^{2}$ to ff scattering. They are the diagrams in fig. $3 a-c$ and fig. $4 a, b$. The former diagrams fall into type 2 above (recall that the $\mathrm{WH} \chi$ vertex is linear in momentum). The latter diagrams are examples of type 1.

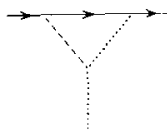

(a)



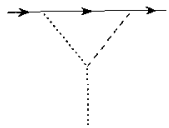

(b)

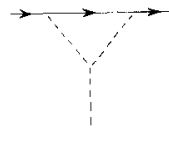

(d)

Fig. 4. Vertex corrections to the $\chi \mathrm{f} \overline{\mathrm{f}}$ and $\mathrm{Hf} \overline{\mathrm{f}}$ vertices. These diagrams contain a three-Higgs vertex; hence the Feynman rules provide a factor of $m_{\mathbf{H}}^{2}$. See fig. 2 for a definition of the various lines. 
These exhaust all possibilities. For example, the diagrams in fig. $4 \mathrm{c}, \mathrm{d}$ fail to contribute because the external physical Higgs eventually show up as $\left(m_{\mathrm{H}}^{2}-t\right)^{-1}$ in ff scattering.

We then calculate the relevant diagrams (for additional details, see ref. [12]). Figs. $3 a-c$ give a leading contribution to the $V+A$ term of the form:

$$
\frac{g^{3} m_{\mathrm{f}}^{2}}{m_{\mathrm{W}}^{2}} \ln m_{\mathrm{H}}^{2} \text {. }
$$

To see the origin of the $\mathrm{V}+\mathrm{A}$ piece, we write down the divergent part of the loop integration of fig. $3 \mathrm{a}$ :

$$
\frac{g^{3} m_{\mathrm{f}}^{2}}{m_{\mathrm{W}}^{2}} \int \mathrm{d}^{n} k \frac{\gamma \cdot k \gamma_{\mu}\left(1+\gamma_{5}\right) \gamma \cdot k}{\left[m_{\mathrm{e}}^{2}+\left(p^{\prime}-p+k\right)^{2}\right]\left[m_{\mathrm{e}}^{2}+k^{2}\right]\left[m_{\mathrm{H}}^{2}+(p-k)^{2}\right]},
$$

(where $p, p^{\prime}$ are the momenta of the ingoing and outgoing fermions). Now, in the dimensional regularization procedure, we make the substitution

$$
k_{\mu} k_{\nu} \rightarrow \frac{1}{n} k^{2} \delta_{\mu \nu}
$$

Hence,

$$
\gamma \cdot k \gamma_{\mu}\left(1+\gamma_{5}\right) \gamma \cdot k=\left(\frac{2}{n}-1\right) \gamma_{\mu}\left(1-\gamma_{5}\right) k^{2}
$$

Thus, this diagram is in fact pure $\mathrm{V}+\mathrm{A}$. When we use these results to calculate ff scattering, we obtain a correction term of the form

$$
\frac{g^{2} s}{m_{\mathrm{W}}^{2}-t}\left[\frac{g^{2} m_{\mathrm{f}}^{2}}{m_{\mathrm{W}}^{2}} \ln m_{\mathrm{H}}^{2}\right]
$$

for $\mathrm{W}$ exchange in the limit of large $m_{\mathrm{H}}$.

We have obtained eq. (5) by neglecting the $\mathrm{V}$ - A piece of the Wff vertex correction. When we calculate the $\mathrm{V}-\mathrm{A}$ piece, we obtain a result (before renormalization) of the form

$$
g^{3}(n-4)^{-1}+\frac{g^{3} m_{\mathrm{f}}^{2}}{m_{\mathrm{W}}^{2}} \ln m_{\mathrm{H}}^{2} .
$$

After renormalization, there are two possibilities. One is that only the $(n-4)^{-1}$ term is cancelled; we then end up with a result similar to eq. (2). The other is that when we perform the subtraction, both terms of eq. (6) are cancelled; leaving no term which grows as $m_{\mathbf{H}}$ gets large. However, the result for the $\mathrm{V}+\mathrm{A}$ piece still stands (since there are no counterterms of a V + A form). Thus, the conclusions of eq. (5) remain unchanged.

We now consider the effect of $\chi$ exchange. Figs. $4 a$, $b$ give a leading contribution of the form

$$
\frac{g^{3} m_{\mathbf{f}}^{3}}{m_{W}^{3}} \ln m_{\mathrm{H}}^{2}
$$


which can be neglected as compared with eq. (2). Thus, the correction term for $\mathrm{W}$ exchange is the dominant correction to ff scattering. The factor of $\left(m_{\mathrm{f}} / m_{\mathrm{W}}\right)^{2}$ arises from the fact that the coupling of the Higgs to the fermions is proportional to $m_{\mathrm{f}} / m_{\mathrm{W}}$.

How general is the factor $\left(m_{\mathrm{f}} / m_{\mathrm{W}}\right)^{2}$ ? It is easy to see that if the Higgs has a nonzero expectation value, then it must couple to fermions with a coupling proportional to the fermion mass. If the Higgs couples to fermions, the only gauge-invariant coupling is of the form $g_{\mathrm{Hff}} \bar{\psi} \psi H$. Now, in the U-gauge, we replace $H$ with $H-\langle H\rangle$. Thus, we end up with a term $g_{\mathrm{Hff}}\langle H\rangle \bar{\psi} \psi$ which contributes to $m_{\mathrm{f}}$. Hence, $g_{\mathrm{H} f \mathrm{f}}$ is proportional to $m_{\mathrm{f}}$. The vacuum expectation value $\langle H\rangle$ also contributes to the mass of the vector bosons. Let the gauge covariant derivative be $\mathrm{D}_{\mu}=\partial_{\mu}-i g T^{a} W_{\mu}^{a}$ where $W_{\mu}^{a}$ is the W-boson field. Then, the term in the Higgs Lagrangian $\left(\mathrm{D}_{\mu} H\right)^{+}\left(\mathrm{D}_{\mu} H\right)$ leads to $m_{\mathrm{W}} \sim g\langle H\rangle$. Combining this with $m_{\mathrm{f}} \sim g_{\mathrm{Hf}} \overline{\mathrm{f}}\langle H\rangle$ yields $g_{\mathrm{Hf}} \sim g\left(m_{\mathrm{f}} / m_{\mathrm{W}}\right)$.

Consider the case of a more complicated Higgs structure. Then we will find that for one particular Higgs, $\mathrm{H}, m_{\mathrm{f}} \geq g_{\mathrm{Hff}}\langle H\rangle$ and $m_{\mathrm{W}} \geq g\langle H\rangle$ since in general more than one Higgs will contribute to the masses. Thus, the only way to obtain a Higgs-fermion coupling that is not small is to require that $\left.\left.m_{\mathrm{W}}\right\rangle\right\rangle g\langle H\rangle$. In that case, $\langle H\rangle$ can be very small so that $g_{\mathrm{Hf} \overline{\mathrm{f}}} \leq m_{\mathrm{f}} /\langle H\rangle$ can be large. We will consider this possibility in sect. 3.

If we assume that $m_{\mathrm{W}}$ is not much larger than $g\langle H\rangle$, then we are forced to have the factor $\left(m_{\mathrm{f}} / m_{\mathrm{W}}\right)^{2}$ in the one-loop correction to the amplitude for ff scattering. What about the two-loop level? We need a vertex correction where no Higgs bosons touch the fermion line. Such an example is given in fig. 5a. By power counting and our previous analysis, it appears that this diagram yields a contribution proportional to $m_{\mathrm{H}}^{2} \ln m_{\mathrm{H}}^{2}$. (Note that the $H \chi \chi \chi$ vertex provides a factor of $m_{\mathrm{H}}^{2}$ here.) However, if one adds to this the diagram in fig. $5 \mathrm{~b}$, one finds that the term proportional to $m_{\mathrm{H}}^{2} \ln m_{\mathrm{H}}^{2}$ exactly cancels. The remaining pieces do not grow as $m_{\mathrm{H}}^{2} \rightarrow \infty$. We have verified this result in the SU(2) model by analyzing the one-loop graphs for the WW $\chi$ vertex. Note that the $W W \chi$ vertex is absent at the tree level; thus higher-order graphs must be finite. Other two-loop diagrams can behave (at best) as $g^{5} m_{\mathrm{f}}^{2} m_{\mathrm{H}}^{2} / m_{\mathrm{W}}^{4}$ or $g^{5} \ln m_{\mathrm{H}}^{2}$ as $m_{\mathrm{H}} \rightarrow \infty$; hence they will have no observable experimental consequences.

Suppose there is a Higgs boson with zero vacuum expectation value which can couple to fermions. Then, its coupling to fermions is arbitrary. Can this lead to large higher-order corrections as $m_{\mathrm{H}} \rightarrow \infty$, not proportional to $m_{\mathrm{f}}$ ? We conclude again
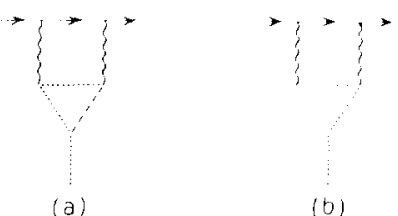

Fig. 5. Two-loop vertex corrections to the $x$ f $\bar{f}$ vertex. We have drawn only two of many possible two-loop corrections. See fig. 2 for a definition of the various lines. 


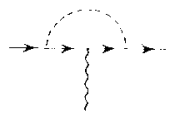

(a)



(b)

Fig. 6. The Wf $\overrightarrow{\hat{f}}$ vertex correction when $\langle H\rangle=0$. If the Higgs boson has zero vacuum expectation value, then figs. $3 b, c, e, g, h$, and $i$ are replaced by diagrams (a) and (b) above.

that there are no such corrections. The reason is that a Higgs with $\langle H\rangle=0$ does not give mass to the W's. Thus, in the absence of all other Higgs, the resulting theory would be a massless Yang-Mills theory coupled to scalar particles. Provided that this coupling is gauge invariant, such a theory is renormalizable. Therefore, the Higgs mass does not play the role of a cutoff for an unrenormalizable theory.

We can verify this explicitly in the SU(2) model by calculating the two diagrams in fig. 6 in the limit of $m_{\mathrm{H}} \rightarrow \infty$. We see that because of the gauge invariant coupling of the Higgs to the W's, the terms proportional to $\ln m_{\mathrm{H}}^{2}$ in fig. 6 a exactly cancel those in fig. $6 \mathrm{~b}$. Note that if $\langle H\rangle \neq 0$, then the Hff $\bar{f}$ coupling is not the same as the $\chi \mathrm{f} \overline{\mathrm{f}}$ coupling; so that such a cancellation does not occur.

We have found that in any gauge theory, only Higgs particles such that $\langle H\rangle \neq 0$ could lead to large observable higher-order corrections. But, in such cases, we have an extra suppression of $\left(m_{\mathrm{f}} / m_{\mathrm{W}}\right)^{2}$ to ff scattering. Furthermore, at the one-loop level, the correction is proportional to $\ln m_{\mathrm{H}}^{2}$ rather than $m_{\mathrm{H}}^{2} / m_{\mathrm{W}}^{2}$. We conclude that in conventional gauge theories (where $g_{\mathrm{Hff}} \overrightarrow{\mathrm{f}}$ is expected to be small) observable weak cross sections will not get large at high energies.

\section{A way to get large effects}

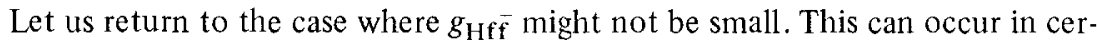
tain gauge theories [5-7] where $\left.\left.m_{\mathrm{W}}\right\rangle\right\rangle g\langle H\rangle$ for one of the Higgs with non-zero vacuum expectation value. As an example, we point to a model of Deshpande and Ma [7]. They investigated an $\mathrm{SU}(2) \times \mathrm{U}(1)$ model with two Higgs multiplets. In their model there were two vacuum expectation values $V_{1}$ and $V_{2}$ and $m_{\mathrm{W}} \sim g\left(V_{1}^{2}\right.$ $\left.+V_{2}^{2}\right)^{1 / 2}$. They found that if $\kappa \equiv m_{\mathrm{Z}}^{2} \cos ^{2} \theta_{\mathrm{W}} / m_{\mathrm{W}}^{2}$, then

$$
\frac{V_{2}}{V_{1}}=\left(\frac{1-k}{\kappa}\right)^{1 / 2} \text {. }
$$

Thus, for $\kappa$ close to 1 (as suggested by the data ${ }^{\star}$ ), $V_{2}<<V_{1}$. Therefore, it follows that $m_{\mathrm{W}}>\mathrm{gV} V_{2}$ as desired. We conclude that models exist where $g_{\mathrm{Hff}} \overline{\mathrm{f}}$ need not be small. It seems plausible that a large Higgs mass in such a theory could lead to ob-

* L. Sehgal [ 13] has obtained $\kappa=0.98 \pm 0.05$ from an analysis of inclusive hadron production in neutrino production. 
servable higher order effects. In fact, recent calculations by Toussaint [14] suggest that in some models with charged Higgs, a large $m_{\mathrm{H}}$ does lead to an observable renormalization of $k$.

Phenomenologically, the situation where $g_{\mathrm{Hf}} \overline{\mathrm{f}}$ is large because $\langle H\rangle$ is small may be constrained. A large $g_{\mathrm{Hf}} \overrightarrow{\mathrm{f}}$ will contribute to many processes $(\kappa, g-2$, ee scattering, etc.). Clearly there is some limit on $g_{\mathrm{Hff}} \bar{f}$ from such data. Combining this with theoretical relations between $g_{\mathrm{Hf}} \overline{\mathrm{f}}$ and $m_{\mathrm{H}}^{2}$, it may be possible to get useful limits; this is currently under investigation.

Another way to increase the value of the suppression factor $m_{\mathrm{f}} / m_{\mathrm{W}}$ is to consider including heavy fermions as intermediate states in loops [15]. It is interesting to note that for $m_{\mathrm{f}} \geq m_{\mathrm{W}}$ one obtains large couplings, so perturbative arguments might be expected to break down for fermion masses lighter than the $1 \mathrm{TeV}$ required in the boson sector. Similarly, quark and lepton widths get large for $m_{\mathrm{f}} \sim m_{\mathrm{W}}$ since they grow as $G_{\mathrm{F}}^{2} m_{\mathrm{f}}^{4}$ (from the weak matrix element squared), which at least raises questions concerning the meaning of point particles or confined quarks with lifetimes of $10^{-23} \mathrm{~s}$. Whether there is a relation between these two effects for $m_{\mathrm{f}} \geq m_{\mathrm{W}}$ is not clear to us.

We can summarize the situation by saying that in gauge theories with one Higgs there should be no observable dynamical effects at very high energies. Theories can be written, with two or more Higgs multiplets, which can give large effects not due to W's and Z's at very high energies, but it is not yet clear whether any of them are consistent with other constraints required by the data.

\section{Production of two W's}

Producing two intermediate vector bosons from a f $\bar{f}$ pair is easily done kinematically with expected $\bar{p}$ p or pp colliding beams, and gives a cross section that grows with energy (for longitudinal W's) in a non-renormalizable theory. Might we expect a large rate experimentally at high energies due to a large Higgs mass which could be studied to help test gauge theories beyond the $W, Z$ level? Again, unfortunately, the fermion mass factor enters and makes the Higgs contribution negligible.

This comes about as follows. At the tree level the diagrams of figs. 1a-d contribute. As is well-known $[4,8,9]$, the $Z^{0}$ (neutral current) contribution (c) provides a cancellation * of the term growing as $s$ from (a) and (b). But (a), (b), and (c) still leave a piece of order $\sqrt{ } s$, which is cancelled by the Higgs boson contribution of $(d)$. After the cancellation in the term of order $s$, the amplitude is approximately

$$
M \sim \frac{m_{\mathrm{f}}}{m_{\mathrm{W}}^{2}} G_{\mathrm{F}} m_{\mathrm{W}}^{2} \overline{v u}\left(1+\frac{s}{m_{\mathrm{H}}^{2}-s}\right) .
$$

\footnotetext{
* Note that these calculations are done in the U-gauge.
} 
Since $\bar{v} u \sim \sqrt{ } s$ as $s \rightarrow \infty$, this falls as $m_{\mathrm{H}}^{2} / \sqrt{ } s$, but without the cancellation it would grow as $\sqrt{ } s$, violating unitarity. The factor of $m_{\mathrm{f}}$ comes from the Higgs coupling in (d) and from the propagator or use of the Dirac equation on the spinors from the other contributions.

This is clearly small because of the factor $m_{\mathrm{f}} / m_{\mathrm{W}}$. It could only get large for $m_{\mathrm{H}}^{2}$ large and $s \simeq m_{\mathrm{H}}^{2}$. In that case

$$
1 /\left(m_{\mathrm{H}}^{2}-s\right) \rightarrow 1 /\left(m_{\mathrm{H}}^{2}-s-i m_{\mathrm{H}} \Gamma_{\mathrm{H}}\right),
$$

giving a cross section (put $s \simeq m_{\mathrm{H}}^{2}$ )

$$
\sigma \sim G_{\mathrm{F}}^{2} m_{\mathrm{f}}^{2} m_{\mathrm{H}}^{2} / \Gamma_{\mathrm{H}}^{2} .
$$

For comparison, one can calculate the contribution to $\sigma$ which is not proportional to $m_{\mathrm{f}}$. This is identical to calculating the cross section for $\nu \bar{\nu} \rightarrow \mathrm{W}^{+} \mathrm{W}^{-}$. In the Weinberg-Salam theory, only diagrams (a) and (c) of fig. 1 contribute [8] (since the neutrino is massless, it does not couple to the Higgs bosons). We then find

$$
\sigma \sim G_{\mathrm{F}}^{2} m_{\mathrm{W}}^{2},
$$

which clearly dominates the contribution of eq. (11).

Experimentally the rate for $\mathrm{e}^{+} \mathrm{e}^{-} \rightarrow \mathrm{W}^{+} \mathrm{W}^{-}$will peak somewhat above threshold and then fall monotonically. The calculations ${ }^{\star}$ in the threshold region give rates that depend on the degree of cancellation of the terms of order $s$, and have been done without the numerically small terms proportional to $m_{\mathrm{e}}$. Fortunately, the cross section is fairly large at its maximum so this is a good way to produce W's, although apparently not a good way to test the dynamical structure of the gauge theories from their high-energy behavior.

\section{Discussion}

Our hope in studying this problem was to find that at very high energies such as $\frac{1}{2} \mathrm{TeV}$ in qq or $\bar{q} \mathrm{q} \mathrm{c} . \mathrm{m}$., which will be achievably with the next generation colliding beam machines, we could expect observable weak interaction effects which would test the dynamics of gauge theories beyond the vector boson sector. We have found that in gauge theories with one Higgs boson such effects are suppressed by a factor $\left(m_{\mathrm{f}} / m_{\mathrm{W}}\right)^{2} \sim 10^{-6}$ and should be quite unobservable.

It is possible to imagine models where large weak effects could occur. There must be more than one Higgs boson, and Higgs particles which have large vacuum expectation values to give mass to vector bosons must not contribute to the masses of the light fermions $\mathrm{e}^{ \pm}, \mathrm{u}, \mathrm{d}$. Higgs particles which couple to the fermions must have vacuum expectation values of the order of the fermion masses but must have large masses (of order $\frac{1}{2} \mathrm{TeV}$ or more) themselves. It may be that such a situation

\footnotetext{
* For a review article containing calculations and further references, see ref. [16].
} 
can be ruled out phenomenologically or theoretically. In particular, present evidence on $\mu$ to e transitions, neutron electric dipole moments, and the strength of the neutral current $m_{\mathrm{W}} / m_{\mathrm{Z}} \cos \theta_{\mathrm{W}}$, all remain consistent, as experiments have improved, with a world in which there is only one Higgs doublet, making more complicated models seem increasingly unlikely.

When high-energy collidng beams are available it will be important to look * for unexpected weak effects to test present ideas about gauge theories. If no effects are found it will be further confirmation of the present standard model. If the present ideas are not completely correct, such high energy effects may show the way to needed improvements.

We appreciate helpful discussions with York-Peng Yao, J. Ellis, R. Cahn, M. Einhorn, F. Wilczek and B. Weeks.

\section{References}

[1] M. Veltman, Acta Phys. Pol. B8 (1977) 475.

[2] M. Veltman, Phys. Lett. 70B (1977) 253.

[3] B.W. Lee, C. Quigg and H.B. Thacker, Phys. Rev. D16 (1977) 1519; Phys. Rev. Lett. 38 (1977) 883.

[4] D.A. Dicus and V.S. Mathur, Phys. Rev. D7 (1973) 3111.

[5] Duane A. Dicus, Vigdor L. Teplitz and J.E. Young, Phys. Rev. D11 (1975) 3295.

[6] D.A. Ross and M. Veltman, Nucl. Phys. B95 (1975) 135.

[7] Nilendra G. Deshpande and Ernest Ma, Phys. Rev. D15 (1977) 1950.

[8] S. Weinberg, Phys. Rev. Lett. 27 (1971) 1688.

[9] C.H. Llewellyn-Smith, Phys. Lett. 46B (1973) 233.

[10] S. Weinberg, Phys. Rev. Lett. 19 (1967) 1264;

A. Salam, in Elementary particle theory, ed. N. Svartholm (Almquist and Wiksell, Stockholm, 1968) p. 367.

[11] G. 't Hooft and M. Veltman, Nucl. Phys. B44 (1972) 189.

[12] H.E. Haber, Doctoral thesis, University of Michigan (1978), unpublished.

[13] L. Sehgal, Invited talk at the Neutrinos 78 Conf., Purdue University, April, 1978.

[14] D. Toussaint, Princeton University preprint (1978).

[15] M. Chanowitz, M. Furman and I. Hinchliffe, LBL preprint in preparation (1978).

[16] J. Ellis and M.K. Gaillard, in Physics with very high energy $\mathrm{e}^{+} \mathrm{e}^{-}$colliding beams, CERN $76-18(1976)$.

[17] H.E. Haber and G.L. Kane, preprint UM HE 78-11 (1978), Nucl. Phys. B, in press.

\footnotetext{
* We have recently discussed in detail the possibility that parity-violating effects signalling weak interactions can be studied at high energies and large $p_{\mathrm{T}}$ by detecting hadron resonance decays and $\Lambda$ 's. Such methods should allow one to detect $W$ 's and $Z$ 's and other "weak" cross sections, and to establish that they are weak interaction effects. See ref. [17].
} 